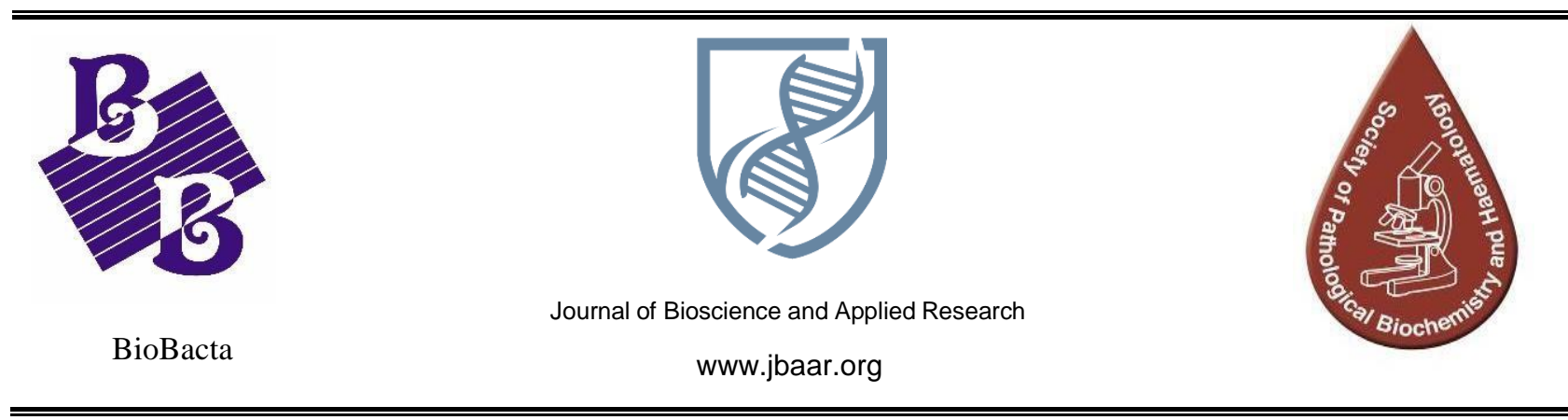

\title{
Nephroprotective Effect of Melatonin against Aluminum Phosphide Induced Renal Tissue Damage in Rats
}

\author{
Mohamed SA. El-Gerbed \\ Zoology Department, Faculty of Science, Damenhour University, Damenhour, Egypt \\ Corresponding author: Mohamed Saad Allah El-Gerbed \\ (https://orcid.org/0000-0001-5126-0353). \\ E-mail: m.elgerbed@yahoo.com
}

\begin{abstract}
Aluminum phosphide (AlP) is a cheap, effective and commonly used agricultural pesticide. The present experiment was undertaken to investigate the effect of melatonin against aluminum phosphide -induced renal toxicity in rats. Forty male rats were divided into four groups. Group I: rats maintained as control, group II: rats received melatonin, group III: rats received AlP, group IV: rats received AlP and melatonin with same previous doses. Data showed that AIP "GIII" treatment resulted in a significant increase in serum creatinine and urea level. Also, a markedly significant increase in lipid peroxidation (MDA). On the other hand, the administration of AlP causes a significant decrease in enzymatic antioxidants activities (superoxide dismutase, catalase, glutathione peroxidase and glutathione reeducates) in the kidney. The histopathological examinationof kidney of AlP -treated group rats, revealed kidney injury with necrotic changes, enlargement of many glomeruli, tubular dilatation and leukocytic infiltration. Electron micrographs of the renal corpuscle showed obvious signs of injury, focal segmental thickening, and podocyte changes, mesangial cells appeared highly deteriorated. Also, proximal convoluted tubules lining cells revealed tremendous alterations and abnormalities in architectural features, an increasing number of the irregular shape of mitochondria with fragmented cristae. Moreover, kidney tissues showed markedly higher p53 induction. Additionally, treatment with melatonin alleviates the nephrotoxicity of AlP by significantly renormalize the Serum enzymatic and biochemical parameters. The biochemical results were supported by histopathological and ultrastructural observations of the kidney. Moreover,inhibition of p53 with melatonin was seen. From these results, it could suggest that the melatonin might be usefulfor preventing nephrotoxicity caused by aluminum phosphide through ameliorative effects on (especially parameters) biochemical indices, oxidative stress, histological and ultrastructural changes.
\end{abstract}

Keywords: Aluminum phosphide, nephrotoxicity, melatonin, histopathological, ultrastructural, Oxidative stress 


\section{Introduction:}

Providing food has always been a challenge to human; the only major cornerstone in this challenge is the competition with pests (Gunnell, Eddleston et al. 2007). Different kinds of pests including animals and insects are the major problem in agriculture, hygiene, and industry (Eddleston, Karalliedde et al. 2002).

"Wheat pill" aluminum phosphide (ALP) (also known as rice tablet) is a colorless and flammable pesticide that excessively used as an insecticide, rodenticide and commonly used fumigant pesticide that is used in the protects stored grains, maintaining processed foods and agricultural products, it is effective, cheap, and easily-available (Yousef, Soliman et al. 2015).

ALP is a toxic and lethal solid pesticide to both human beings and animals (Dua, Kumar et al. 2010). The toxicity is due to deadly phosphine (hydrogen phosphide, phosphorus trihydride or $\mathrm{PH}_{3}$ ) gas released when it reacts with water, presence of moisture in the air or hydrochloric acid in the stomach. Phosphine is a cytotoxic compound that is rapidly absorbed by inhalation, dermally, gastrointestinal (Rahbar Taramsari, Badsar et al. 2013), causes free radicalmediated injury, inhibits vital cellular enzymes and is directly corrosive to tissues (Sudakin 2005, Mehrpour, Jafarzadeh et al. 2012).

ALP toxicity is potentially deadly and affects multiple systems with dangerous consequences. Its toxic effects are displayed in different forms including neurotoxicity (Tripathi and Pandey 2007), electrolyte imbalance and metabolic disturbances (Anand, Binukumar et al. 2011), hepatotoxicity, (Moghadamnia 2012), hematological toxicity (Khurana, Gambhir et al. 2009), cardiotoxicity, (Anand, Binukumar et al. 2011), 2011) and nephrotoxicity, (Gurjar, Baronia et al. 2011).
Nature produces a group of antioxidants that prevent the production of free radicals and protect against oxidative damage caused by various pesticides Recently, melatonin (N-acetyl-5-methoxytryptamine), a neurotransmitter and major pineal gland's secretory product, and has been shown to be an effective antioxidant and free radical scavenger. (Bhatti, Kiran et al. 2010).

Melatonin (MEL), as an amphiphilic molecule, crosses all morphophysiological barriers and can be found particularly in mitochondria, protecting them from oxidative stress (Govender, Loos et al. 2014). The melatonin protective actions have been previously notified against various pesticide and metal toxicities (Romero, Ramos et al. 2014, Asghari, Moloudizargari et al. 2017). Additionally, Melatonin elevates the performance of antioxidant enzymes such as GPx, GSH, SOD, and CAT. Also, MEL has been shown to play a vital role by lowering the standards of oxidative stress parameters such as protein carbonyl (PC) content malondialdehyde (MDA) activity and myeloperoxidase (MPO) activity (Kolli, Abraham et al. 2013). Moreover, it has been elucidated that MEL exerts its effectiveness by way of stimulation of nuclear factor erythroid 2-related factor 2 (Nrf2) and antioxidant enzyme heme oxygenase-1 (HO-1) (Kilic, Kilic et al. 2013). Also, MEL exhibits anti-inflammatory effects by reducing the expression of COX-2 (Ahmadiasl, Banaei et al. 2014). Finally, we assessed the possible protective effects of melatonin against aluminum phosphide -induced renal toxicity in rats, through reducing oxidative stress and, decreased and inhibit functional and structural impairment in rat kidney.

\section{Materials and Methods}

\section{Chemicals:}

Aluminum phosphide ( $>95 \%$ purity) was from Chem Service (West Chester, PA, USA). The molecular weight of this compound is $57.955 \mathrm{~g} / \mathrm{mol}$ with the molecular formula of AlP. It is obtainable as a tablet of 
$3 \mathrm{~g}$ that contains $44 \%$ ammonium carbonate and $56 \%$ Alp. Melatonin was from Sigma Chemical Co. (St Louis, MO, USA). Immunohistochemical assay kits were purchased from Lab Vision (Thermo scientific Immunohistochemistry Solutions). All other chemicals used were of the highest purity and analytical grade purchased from commercial suppliers.

\section{Animals and experimental protocol:}

The investigation was performed on only forty healthy male adults sexually mature, albino rats weighing $(140 \pm 5 \mathrm{~g})$ were purchased from the Laboratory Animal Breeding Colony of the National Research Centre, Dokki Giza, Egypt., were used for the study. They were acclimated to conditions for 1 week before the experiment. The animals were housed in plastic cages under natural room temperature $\left(26 \pm 2^{\circ} \mathrm{C}\right)$ and lighting conditions. Rats were fed with basal commercial laboratory diet and drinking distilled water was given through drinking bottles throughout the experiment. The rats were weighed weekly during the experimental period. The experiments were approved by the Animal Ethics Committee of Faculty of Science, Damenhour University, Damenhour, Egypt. Rats were randomly assigned into four groups 10 animals each and were treated as follows:

The Control group (GI): Rats served as normal control (orally received $1 \mathrm{ml} / \mathrm{kg}$ body weight of corn oil).

Melatonin group (GII): Rats received orally melatonin (10mg/kg b.w.) according to (Bhatti, Sidhu et al. 2014). Melatonin was dissolved in corn oil.

Aluminum phosphide group (GIII): Rats treated with $\mathrm{AlP}$ at a dose of $1.15 \mathrm{mg} / \mathrm{kg}$ b.w. (one-tenth LD50) in corn oil (Bingham, Cohrssen et al. 2001).

AlP + MEL group (GIV): Rats were given AlP and then they were given Melatonin (with the same previous dosage received by the rats in groups III and II, respectively).

\section{Blood collection and tissue preparation:}

At the end of the experimental period (thirty days), rats of all rats were kept fasting for 12 hours then sacrificed under chloroform anesthesia. The blood samples were withdrawn from the heart in plain tubes and left to clot, then centrifuged for 10 minutes at $3000 \mathrm{rpm}$ to obtain clear sera. Sera were stored at $20^{\circ} \mathrm{C}$ and used to determine the activities of the Creatinine and urea concentrations. To further verify the injury of the kidney, slices were immediately removed; washed using cold physiological saline solution, then used for various lipid peroxidation, antioxidant enzymes, Histopathological and p53 expression examinations. The remaining kidney tissue was immersed into $2.5 \%$ glutaraldehyde for ultrastructural studies.

\section{Clinical observations and measurement of bodyweight:}

Each rat was observed daily throughout the experiment period, to determine mortality and any changes in animal behavior. During the treatment period, the bodyweight of each rat was monitored weekly and fasted weights at necropsy.

\section{Assessment of renal function:}

Freshly separated serum was used for estimation of the level of urea and creatinine as kidney function marker by the commercially available diagnostic kits, creatinine (Brod and Sirota 1948), Urea (Tabacco, Meiattini et al. 1979).

\section{Lipid Peroxidation as degraded product Malondialdehyde (MDA):}

The concentration of MDA in kidney tissue, an index of lipid peroxidation, was determined spectrophotometrically according to (Draper and Hadley 1990). 


\section{Antioxidant defense system assays:}

Kidney tissues were minced and homogenized (10\%, w/v), separately, in ice-cold $1.15 \% \mathrm{KCl}-0.01 \mathrm{M}$ sodium, potassium phosphate buffer $(\mathrm{pH}$ 7.4) in a Potter-Elvehjem type homogenizer. The homogenate was centrifuged at $10,000 \mathrm{x}$ g for $20 \mathrm{~min}$ at $4{ }^{\circ} \mathrm{C}$, and the resultant supernatant was stored at $-80{ }^{\circ} \mathrm{C}$ till analysis. Stored supernatant was analyzed for the activities of antioxidant enzymes. Glutathione peroxidase (GPx) and glutathione reductase (GR) activities were assayed according to (Paglia \&Valentine, 1967) and (Goldberg \& Spooner, 1983) respectively. Superoxide dismutase (SOD) activity was evaluated as described by (Marklund and Marklund 1974). Also, CAT activity was determined in supernatant according to (Sinha1972).

\section{Histopathological examinations:}

Histopathological analysis was performed using the standard laboratory procedure. A small piece of the kidney was removed from each rat, washed with saline to remove the blood and then fixed in formalin $(10 \%$ formaldehyde). Dehydration treatment with a serious of different concentrations of ethanol and embedded in paraffin wax. Sections of $4 \mu \mathrm{m}$ thick were performed in rotary microtome Leitz 1512 (Leitz, Wetzlar, Germany). They were double-stained with hematoxylin-eosin (H\&E) as described by (Strate, Mann et al. 2005). To evaluate the histopathological changes (Bancroft, Cook et al. 1994), Kidneys were assigned an injury score of ((+) Mild, (++) Moderate; (+++) severe; (-) no pathological Change) to compare the severity of tissue damage.

\section{Ultrastructural study:}

kidney tissues, $1 \mathrm{~mm}^{3}$ thickness, from rats of all groups were fixed in ${ }_{4} \mathrm{~F}_{1} \mathrm{G}$ for 24 hours, then rinsed in phosphate buffer solution $\mathrm{pH}=7.4$ at $4^{\circ} \mathrm{C}$ for 3 hours. Specimens were then post fixed in $1 \%$ osmium tertraoxide at $4^{\circ} \mathrm{C}$ for $1-2$ hours, and then they were washed with $0.1 \mathrm{M}$ phosphate buffer $(\mathrm{pH}=7.2)$ repeatedly for 10 minutes. Specimens were dehydrated in ascending graded ethanol series followed by propylene oxide. Polymerization and embedding were carried out by using the AralditeEpon mixture in an oven at $58^{\circ} \mathrm{C}$. Semithin $(1 \mu \mathrm{m})$ and ultrathin $(50 \mathrm{~nm})$ sections were cut on LKB-III ultramicrotome. The ultrathin sections were picked up on 200 naked copper grids. They were double-stained with uranyl acetate for 30 minutes and then with lead citrate for 20-30 minutes.

35 Grids were examined with a Jeol 100 CX transmission electron microscope, Faculty of Science, Alexandria University, Egypt.

\section{Detection of p53 by immunohistochemistry:}

Ten kidney sections from each group were randomly fixed in $10 \%$ neutral formalin. Following fixation, specimens were dehydrated, embedded in wax_and sectioned in $4-\mu \mathrm{m}$. The tissue sections were cut 1-2 days prior to immunostaining to avoid potential problems in antigen recognition due to storage degradation of the cut tissue sections on glass slides (Prioleau and Schnitt 1995). Sections were transferred to a $10 \mathrm{mmol} / \mathrm{l}$ citrate buffer solution at a $\mathrm{pH}$ of 6.0 and heated at $80 \mathrm{C}$ for $30 \mathrm{~min}$ in the microwave to restore antigens for p53staining. After washing in water, 3.0\% $\mathrm{H}_{2} \mathrm{O}_{2}$ in methanol was applied for $20 \mathrm{~min}$, in order to block endogenous peroxidase activity. The intensity of p53 expression in positively stained cells (7-10 microscopic fields from well-labeled areas were randomly used at $\times 200$ magnification) was analyzed on Intel ${ }^{\circledR}$ Core ${ }^{1}{ }^{\circledR}$ based computer using Video Test Morphology® software (Russia) with a specific built- in routine for area, \% area measurement and object counting.

\section{Statistical Analysis:}

Data are given as mean \pm SE of ten animals in each group. Statistical analysis was performed by one-way analysis of variance (ANOVA). Significant differences between means were determined by the level of significance was $\mathrm{P}<0.05$. 


\section{Results:}

\section{Clinical findings:}

During the experiment (30 days of treatment), noneof the rats in all groups died. Rats of both control group (GI), and melatonin (GII), exhibited good health with no obvious abnormal appearance and behavior. On the other hand, rats received ALP treated groups (GIII), revealed, loss of appetite, reduced physical activity andslight diarrhea. In addition, Rats receiving ALP and melatonin (GIV), did not show any specific behavioral changes and abnormalities in the external features. Also, body weight, was found to be stable, did not differ during the experiment between the control group (GI) and the melatonin - treated group (GII). Body weight had decreased in the ALP treated groups (GIII), as compared to that of the control group. On the other hand, the administration of melatonin to ALP rats (GIV), preserved body weight, increased were observed, when compared to the ALP group, by the endof the experiment.

\section{Effect of melatonin on serum renal markers:}

The measured concentration of creatinine and urea in the blood serum to monitor the toxic effect of ALP and the preventive role of melatonin. No significant difference was a notice in the melatonin alone group (GII) as compared to that of the control group (GI) $(\mathrm{P}>0.05)$. The Creatinine and urea concentrations were significantly $(\mathrm{P}<0.05)$ increased in the ALP-treated group (GIII), as compared to that of the control group. While rats receiving both melatonin and ALP (GIV), represented significant $(\mathrm{P}<0.05)$ reduction in the levels of these parameters as compared to that of ALP group (GIII) (Table 1).

Table 1. Effect of melatonin treatment on serum nephrotoxicity indices induced by aluminum phosphide in rats.

\begin{tabular}{lllll}
\hline \multirow{2}{*}{ Parameters } & \multicolumn{2}{l}{$\begin{array}{l}\text { Treatment } \\
(\text { Dose } / \mathrm{kg})\end{array}$} & & \\
\cline { 2 - 5 } & Control & melatonin & ALP & ALP/ melatonin \\
\hline Creatinine $(\mathrm{mg} / \mathrm{dl})$ & $0.72 \pm 0.13$ & $0.82 \pm 0.12 \#$ & $1.83 \pm 0.52^{*}$ & $1.25 \pm 0.18^{*}, \#$ \\
Urea $(\mathrm{mg} / \mathrm{dl})$ & $63.68 \pm 2.51$ & $68.75 \pm 2.57 \#$ & $127.81 \pm 13.26^{*}$ & $72.33 \pm 4.74^{\#}$ \\
\hline
\end{tabular}

ALP: aluminum phosphide

Data are expressed as mean \pm SE for eight rats in each group.

* Indicates a significant difference from control at $P<0.05$ by one-way ANOVA.

${ }^{\#}$ Indicates a significant difference from ALP at $P<0.05$ by one-way ANOVA. 
Effect of melatonin on lipid peroxidation (LPO) and enzymatic antioxidants status:

MDA levels and activities of antioxidant enzymes in the kidney of all groups are represented in Table 2. No significant difference $(\mathrm{P}>0.05)$ in levels of MDA and the activities of SOD, CAT, GPx and, GR between the control group (GI) and melatonin (GII). On the other hand, the MDA level significantly $(\mathrm{P}<0.05)$ increased in the ALP group (GIII) as compared to that of a control group (GI). In addition, administration with melatonin caused significant $(\mathrm{P}<0.05)$ decrease in the levels of MDA when compared to ALP-treated rats and improved the elevation of kidney LPO to reach the control level. In contrast, a significant $(\mathrm{P}<0.05)$ reduction in the activities of the enzymatic antioxidants (SOD, CAT, and GPx) in ALP received rats (GIII) were observed, except for glutathione reductase (GR) which display insignificant $(\mathrm{P}>0.05)$ decrease. Administration of melatonin prevented ALP elicited a decrease in the activities of these antioxidant enzymes and preserve the activities of SOD and GPX as compared to that of control. SOD and GPx activities exhibited an insignificant difference between the control group (GI) and in ALP plus melatonin group (GIV). While rats receiving ALP and melatonin (GIV), the activity of CAT was increased significantly $(\mathrm{P}<0.05)$ as compared with that of the control group (GI). Remarkably, neither ALP nor melatonin at any treatment caused a significant change in the activities of GR in the kidney. Comparing with the control group, the reductions were seen in GR activity after ALP treatment either alone or in conjunction with melatonin did not reach a statistical significance.

Table 2. Effect of melatonin on ALP-induced alterations in the lipid peroxidation, activities of enzymatic antioxidants and glutathione metabolizing enzymes in kidney of control and experimental rats.

\begin{tabular}{lllll}
\hline Parameter & \multicolumn{2}{l}{$\begin{array}{l}\text { Treatment } \\
\text { (Dose/kg) }\end{array}$} & & \\
\cline { 2 - 5 } & Control & melatonin & ALP & $\begin{array}{c}\text { ALP / } \\
\text { melatonin }\end{array}$ \\
& & & & \\
\hline MDA (nmol/g tissue) & $135.57 \pm 9.42$ & $153.83 \pm 11.21 \#$ & $374.74 \pm 25.44^{*}$ & $182.55 \pm 13.82^{\#}$ \\
SOD (U/mg protein) & $17.82 \pm 0.31$ & $16.22 \pm 0.44 \#$ & $12.43 \pm 0.52^{*}$ & $15.71 \pm 0.36^{\#}$ \\
CAT (U/mg protein) & $83.82 \pm 4.05$ & $86.32 \pm 3.16 \#$ & $51.39 \pm 3.84^{*}$ & $68.27 \pm 2.85^{*}, \#$ \\
GPx (mU/mg protein) & $126.16 \pm 15.37$ & $118.75 \pm 13.41 \#$ & $75.17 \pm 8.43^{*}$ & $109.34 \pm 11.41^{\#}$ \\
GR (mU/mg protein) & $81.53 \pm 6.46$ & $79.82 \pm 9.62$ & $76.72 \pm 7.22$ & $73.31 \pm 5.35$ \\
& & & & \\
\hline
\end{tabular}

ALP: aluminum phosphide; MDA: malondialdehyde; SOD: superoxide dismutase; CAT: catalase; GPx: glutathione peroxidase; GR: glutathione reductase.

Data are expressed as mean \pm SE for eight rats in each group.

* Indicates a significant difference from control at $P<0.05$ by one-way ANOVA.

\# Indicates a significant difference from ALP at $P<0.05$ by one-way ANOVA. 


\section{Histopathological results:}

The histological changes in kidneys were presented with corresponding histopathological scores (Table 3) and the photomicrographs of tissue sections are presented in Figure (Figs.1A - F). The renal tissue consisted of an outer cortex and an inner medulla under a thin renal capsule. The kidneys sections from the renal tissues of the control group (GI) and melatonin groups (GII) rats exhibited normal renal tissue architecture, where normal renal corpuscle is composed of tufts of anastomosing capillaries (glomerulus) which is surrounded by a double-walled capsule, tubular epithelium and interstitial tissue were observed (Figs. 1 $\mathrm{A} \& \mathrm{~B})$. Also, the proximal and distal convoluted tubules (PCT \&DCT) are composed of a single layer of a simple cuboidal type of epithelium; distal tubules had wider lumina compared with proximal tubules, no brush border, less eosinophilic cytoplasm, and smaller and flatter cells (Figs.1A\&B).

Table 3. Effect of treatment with melatonin on the renal histopathology scores induced by treatment of rats with Aluminum phosphide

\begin{tabular}{|c|c|c|c|c|}
\hline Groups & Normal & melatonin & ALP & ALP \& melatonin \\
\hline tubular lumen dilation & - & - & +++ & + \\
\hline Tubular necrosis & - & - & ++ & + \\
\hline Tubular degeneration & - & - & +++ & - \\
\hline Epithelium desquamation & - & - & ++ & - \\
\hline Blood vessel congestion & - & - & +++ & + \\
\hline Glomerular congestion & - & - & ++ & - \\
\hline Urinary Space & - & - & ++ & + \\
\hline Leukocyte infiltration & - & - & ++ & + \\
\hline
\end{tabular}

(+) Mild, (++) Moderate; (+++) severe; (-) no pathological Change.

The renal sections with prolonged ALP treatment (GIII): showed more severe morphological damage in the architecture of the basic structural unit with histopathological scoring of (3+). Glomerular damage with abnormal Bowman's capsule, Progressive inflammatory cell infiltrates associated with thickening of the tubular basement membrane (TBM) and Bowman's capsule (Fig. 1C). Renal tubules appeared degenerative changes in the form of vacuolated cytoplasm and lost definition with indistinct cell margins, brush border appeared distorted (Figs1.C\&D).
Increased intertitular spaces were evident. In addition, rats that were given both ALP and melatonin (GIV), revealed a normal histological appearance of normal tubular arrangements and glomerular architectures and normal Bowmen's capsule. With normal blood vessels (Figs. 1E\&F) when compared with the control group (GI), with minimal pathological alterations (histopathological scoring: 1+), as compared to the other treated groups, indicating for the synergetic effect of melatonin in protecting and inhibiting the damage and alterations caused by the AlP. 

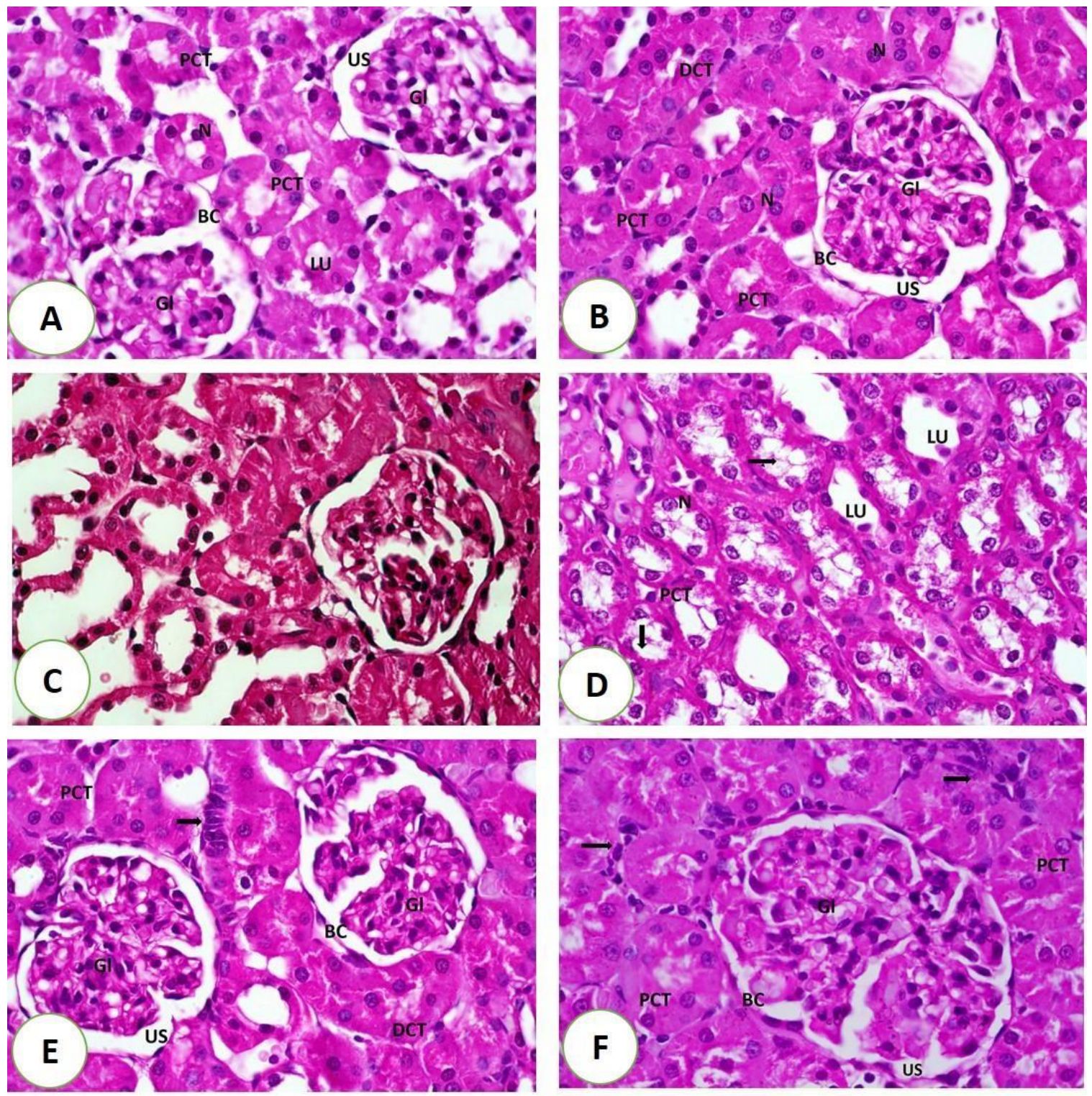

Fig.1(A-F). Photomicrographs of haematoxylin- and eosin-stained sections of the control and treated groups. The control rats and rats treated with melatonin (Figs. A\&B) respectively show normal kidney architecture. Notice: normal Bowman's capsule $(\mathrm{Bc})$, glomerulus tufts $(\mathrm{Gl})$, narrow urinary space (US). Also, proximal convoluted tubules (PCT) with typical cuboidal cells and central round nuclei $(\mathrm{N})$ and narrow lumen $(\mathrm{Lu})$ were observed. $(\mathrm{C} \& \mathrm{D})$ rats received aluminum phosphide demonstrating, kidney tissue with cellular abnormalities, hypertrophied and congested glomerulus (Gl) and dilated urinary space (US). Notice: Tubular necrosis, epithelial lining degeneration, swelling proximal (PCT) and distal (DCT) convoluted tubules with segregated nuclei $(\mathrm{N})$, the dilated lumen $(\mathrm{Lu})$. $(\mathrm{E} \& \mathrm{~F})$ rats receiving aluminum phosphide and melatonin, showing, kidney tissue with typical architecture, normal Bowman's capsule (Bc), glomerulus (Gl), regular urinary space (US). Notice: proximal convoluted tubules $(\mathrm{PCT})$, a narrow lumen $(\mathrm{Lu})$, central rounded nuclei $(\mathrm{N})$. Also, inflammatory cell infiltrates (arrow) were seen. Scale bar: $50 \mathrm{~mm}$. 
Transmission electron microscopic results:

To define ultrastructural changes associated with renal toxicity progression, the kidney glomerular tissues and proximal tubular epithelial cells (PTEC) of all experimental groups of rats were represented in Figures (2A - D). Transmission electron micrographs of sections of control rats (GI) and melatonin groups (GII) rats show a normal glomeruli ultrastructure (Figs.

2 A\&B). The renal corpuscle consists of a tuft of capillaries, the glomerulus, which is invaginated into Bowman's capsule. Podocytes appeared normal with numerous small bell shaped-secondary (foot) processes resting on the outer surface of the glomerular basement membrane. In addition, the interpapillary region of renal corpuscles appeared occupied by a specialized cell type known as mesangial cells. On the other hand, the electron microscopic preparations of kidneys of ALP-treated group rats, (GIII) revealed obvious signs of injury. The glomerular capillaries filled with RBCs appeared highly dilated. Ultrastructural, the endothelial cells that line the glomerular capillaries and rest on an abnormally thickened basement membrane, and the mesangial cells appeared highly deteriorated with condensed heterochromatic nuclei and irregular nuclear envelope. Moreover, the podocytes appeared deformed and destruction the appearance of both primary and secondary processes (Fig. 2C). Also, examinations of electron micrographs of kidney sections of rats that were given both ALP and melatonin (GIV), showed, more or less normal architecture (Fig. 2D), as compared to the control group (GI).

The electron micrograph of control (GI) and melatonin groups (GII) rats revealed that, proximal convoluted tubules with normal ultrastructure (Figs.3A\&B), simple cuboidal epithelial cells rest on a well-defined basement membrane and possess surface specializations. Normal brush border microvilli extending several microns in length with prominent lamina propia. Furthermore, the micrographs showed that the nuclei appeared large oval or rounded in shape and central in location, with chromatin material dispersed within the nucleoplasm and along with a regular nuclear envelope. The nuclear envelope appeared perforated through distinct nuclear pores. Packed pleomorphic mitochondria with electron-dense matrix and parallel to each other and to the longitudinal axis of the cell could be noticed. They were of two shapes, elongated (rod-shaped) mitochondria and rounded ones. Moreover, other cytoplasmic compartments such as free ribosomes, lysosomes and lipid droplets appeared typical in organizations and amounts. 

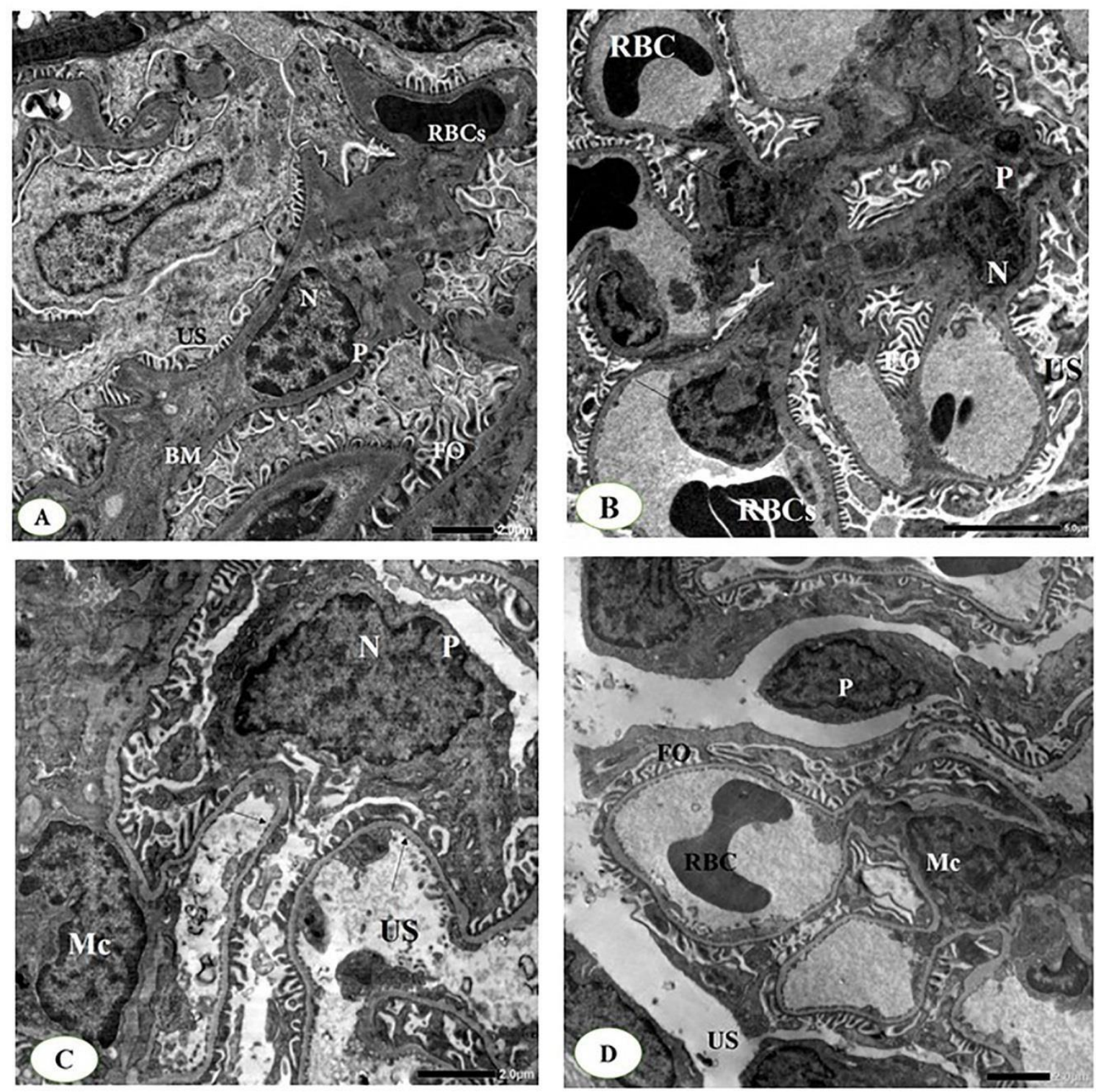

Figure (2 A-D): Transmission electron micrographs of glomerular capillaries sections rat of the control and treated groups. (A\&B) The control rats and rats treated with melatonin respectively, demonstrating normal architecture. Part of the renal corpuscle, distinct urinary space, and podocytes with regularly arranged foot processes with slit pores that are separated from the endothelium by a basal lamina were seen. Note also, Capillary lumen lined with endothelial cells and containing uncompressed red blood cells, mesangial cells. (C) Glomerular capillaries of aluminum phosphide treated rat showing, alteration in the general features, highly dilated glomerulus, mesangial cells injury, the Bowman's space (Bs) appear wide. Moreover, a podocyte exhibits signs of degeneration: the podocytes appeared deformed with irregular nuclei. (D) Rats receiving aluminum phosphide and melatonin showing, kidney tissue with normal architecture, glomeruli showed regular profiles with moderate cellularity. The glomerular capillaries appeared normal. Moreover, the podocytes appeared normal with irregular outline nuclei which appeared normal and podocyte processes appeared normal. 
In the ALP group (GIII), proximal convoluted tubules revealed tremendous alterations and abnormalities in architectural features. The cells showed a failure in cellular differentiation. Brush borders were rare and difficult to detect and fragmented, nuclei shrunk with clumping and margination of nuclear chromatin. The nuclear membranes contained numerous enlarged pores and separated them by dilated spaces. Some nuclei appeared with irregular nuclear envelope, sometimes forming nuclear pockets and contained prominent compact and frequently peripheral segregated nucleoli. Furthermore, examinations revealed that the mitochondria and endoplasmic reticulum appeared highly injured and deformed. There was an increased number of lysosomes, lipid droplets and cytoplasmic vacuoles (Fig. 3C). Moreover, examinations of electron micrographs of kidney sections of rats that were given both ALP and melatonin (GIV), indicated an obvious degree of improvement with normal pattern (Fig. 3D), in comparison with the rats treated with ALP.

\section{Over-expression of $\mathrm{p} 53$ proteins in the renal tissue} following ALP treatment:

The results of the expression of the p53 protein of all experimental groups are shown in figures (4 A - F). Kidney sections of control rats and rats received melatonin group (GII) displayed negligible expression of p53 proteins (Figs.4A, B\& F). However, immunohistochemical staining evaluation kidney of ALP -treated group (GIII) rats, revealing, glomerulus and renal tubular epithelial cells, especially their nuclei, were stained positively with significantly elevated expression of p53 proteins in the kidney tissue. In addition, the expression of $\mathrm{p} 53$ positive tubular cells in the renal cortex and outer medulla layer was relatively higher in this group as compared with that of the control group (Figs.4C, D\& F). On the other hand, screening of kidney sections of rats that received both ALP and melatonin (GIV) showed a significant reduction $(\mathrm{p}<0.05)$ in the positive stained expression of $\mathrm{p} 53$ (Figs.4 E\&F) as compared with that of ALP group (GIII). 

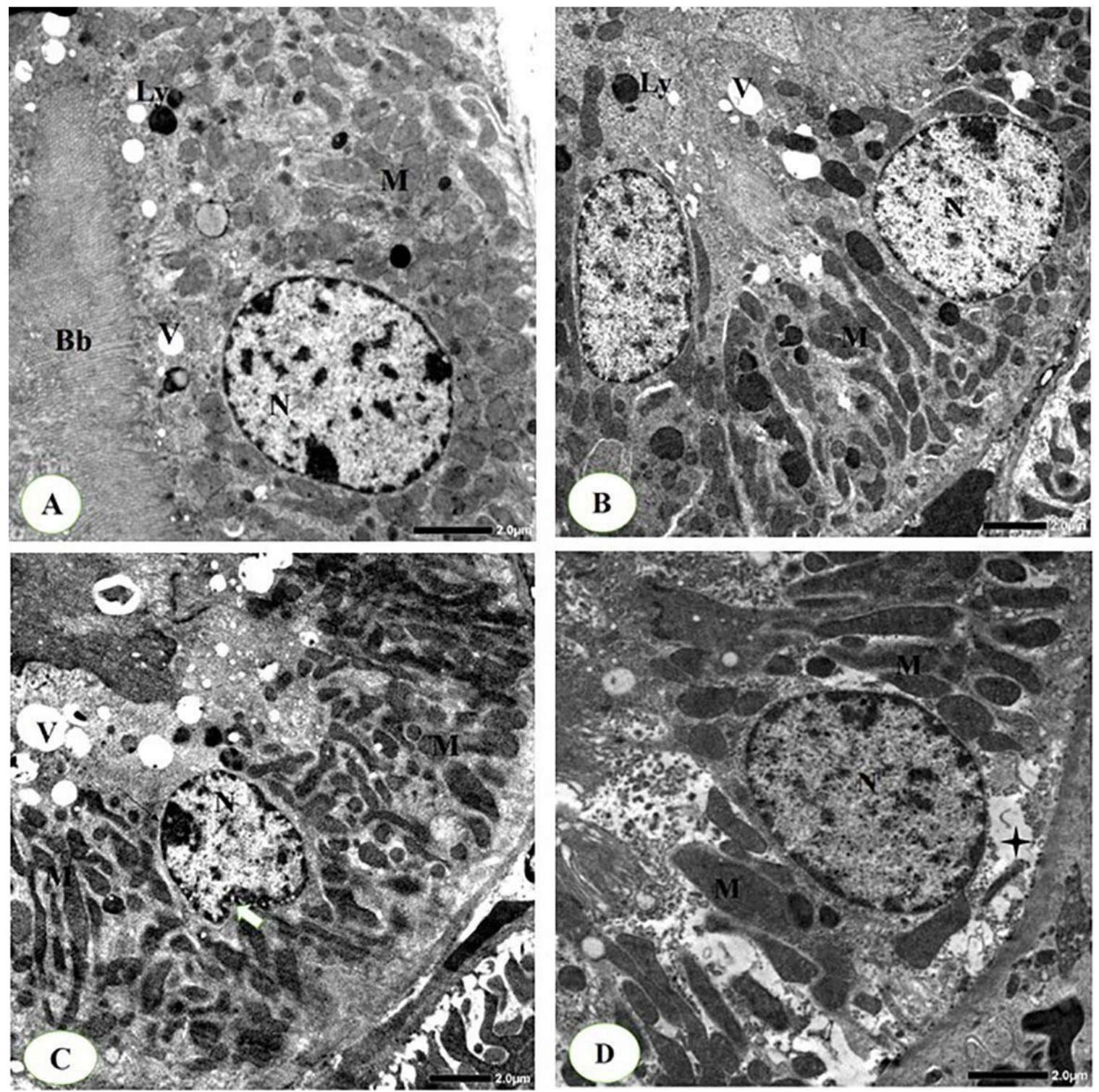

Figure (3 A-D): Transmission electron micrographs of proximal tubular epithelial cells from rats of control and treated groups. (A\&B) the normal architecture of proximal tubular epithelial cells from control and treated rat with melatonin respectively, showing, cuboidal-shaped cells, euchromatic nuclei $(\mathrm{N})$ with the regular nuclear envelope. Note also, numerous electron-dense mitochondria (M), primary lysosome (Ly), well-developed brush border. (C) Proximal tubular epithelial cells from a rat received aluminum phosphide revealed sever alterations and abnormalities in architectural features. Note the necrotic epithelial cells with loss of cytoplasmic organelle and disappearance of brush border, nuclei with chromatin condensed circumferentially having nuclear pockets (white arrow), segregated nucleolus (then arrow). Also, polymorphic mitochondria with loss of cristae, an increase in the number of secondary lysosomes (Ly) and numerous vacuoles (V) were seen. (D) Proximal tubular epithelial cells from rat receiving aluminum phosphide and melatonin showing, the normal architecture of proximal tubular epithelial cells. 

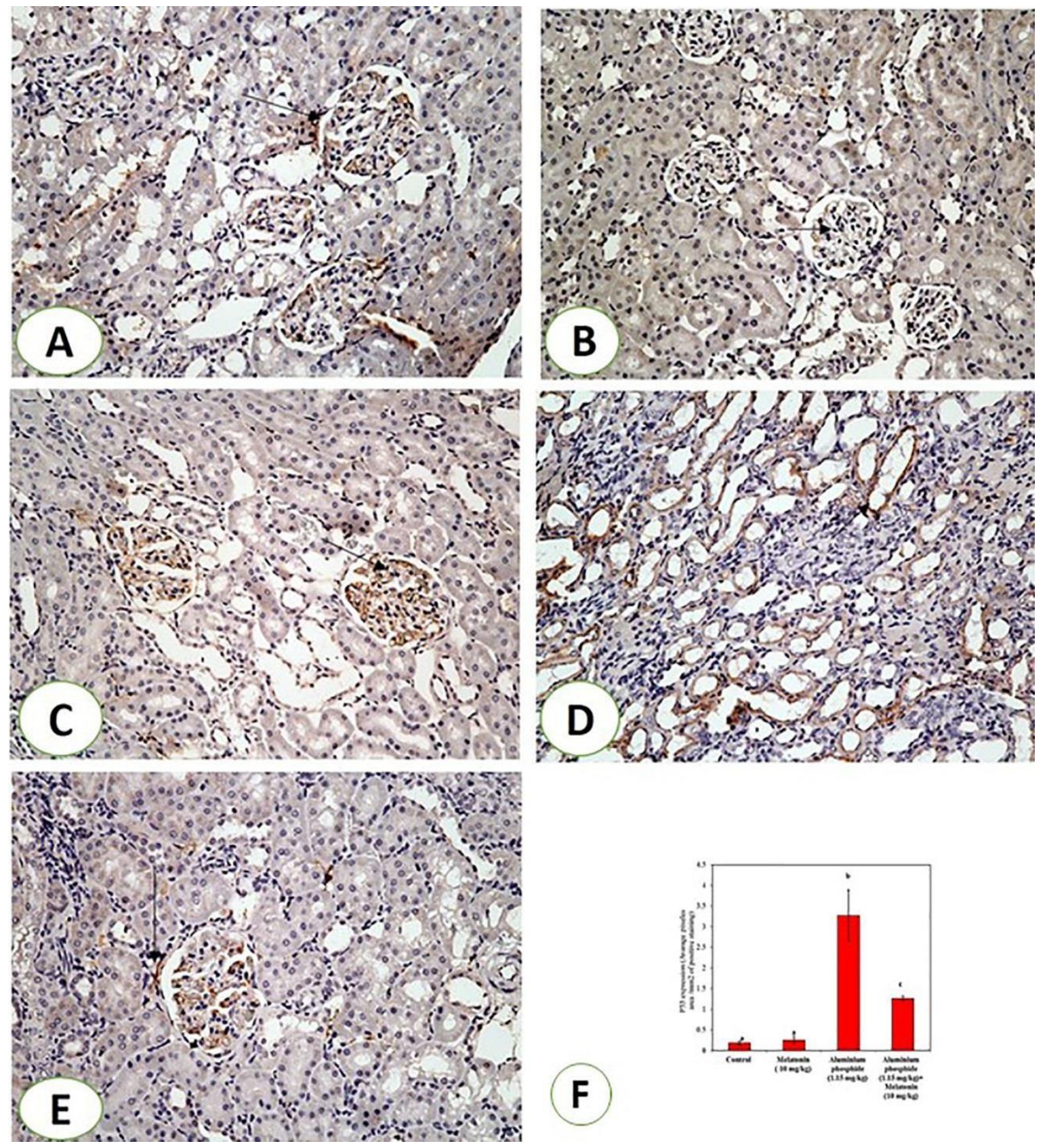

Figure (4 A-F): Immunohistochemical staining for Expression of p53 proteins from rats of control and treated groups. (A\&B) The control rats and rats treated with melatonin respectively, demonstrating, negligible expression of p53 proteins. (C|\&D) Rat received aluminum phosphide revealed strongly p53 expression in renal sections. (E) Rat receiving aluminum phosphide and melatonin showing decreased strongly p53 expression in renal sections. Immunopositively cells (arrows). (F) Expression of p53 in the kidney. The level of P53 expression increased significantly $(\mathrm{P}<0.05)$ in the ALP group compared to the control and MEL groups. P53 expression level significantly decreased $((\mathrm{P}<0.05)$ in the ALP\&MEL group as compared to that of the ALP group. The bars represent \pm SE. of the mean of each group $(n=10)$. Columns superscript with different letters are significantly different, Significance at $\mathrm{P}<0.05$. 


\section{Discussion:}

Pesticides are responsible for Problems related to health in humans such as hypertension, cardiovascular disorders. (Ejaz, Akram et al. 2004). Direct or indirect contact with pesticides leads to neuromuscular defects and stimulation of drug and steroid metabolism (Subramaniam and Solomon 2006). The kidney is one of the major targets of phosphine poisoning in the human body (Sudakin 2005). Therefore, the present study was carried out to examine the renal protective effects of melatonin in ALP toxicity.

In the present study, it was noticed that rats administered ALP, revealed signs of toxicity including, reduced physical activity, loss of appetite and a significant decrease in the mean body weight. The present findings were in general agreement with those stated by Batra et al. (1994) who investigated the oral toxicity of aluminum phosphide in male Wistar rats, he reported that rats exhibited few clinical signs such as reduced activity, increasing weakness anorexia and lack of desire for food, decrease in body weight. Also, the mortality curve was observed, however, during the course of the present study, it was clearly noticed that rats with melatonin appeared more active and healthier with normal consumption of food and water, normal external features and increase in body weight, as compared to that of AlP -treated group. In accordance with their results, (Wolden-Hanson, Mitton et al. 2000) suggested that melatonin administered in drinking water increased the locomotor activity of rats. In accordance with the present data (Detanico, Piato et al. 2009) showed in their study that daily administration of exogenous melatonin singly decreased grooming in mice and reassessed the potential of melatonin as an antidepressant.

Creatinine and urea are two of the classical test signals for kidney functions and renal structural perfection. Data in the present study suggested that aluminum phosphide (GIII), induced intoxication strongly disrupt normal renal markers in rats. significantly $(\mathrm{P}<0.05)$ increased in Creatinine and urea concentrations observed in ALP intoxicated rats were accepted as an indicator of nephrotoxicity and renal injury as compared to that of control. Confirming the present results (Saif, Khan et al. 2015) suggested that an increase in serum blood urea nitrogen has been detected showing renal injury (renal pathology). Serum creatinine elevation is mainly due to impairment in the kidney function through increased turn over in the muscle may also contribute (Parmar, Shah et al. 2009). Data of the present study is in agreement with the results reported previously by (Yousef, Soliman et al. 2015), who studied the efficiency of $\alpha$-lipoic acid in lessening the toxicity of aluminum phosphide on biochemical parameters, free radicals and enzyme activities in serum, liver, and kidney of male rats. These reports have recommended that the overproduction of ROS diminishes the renal functions, which is attached to increases in serum creatinine and urea levels.

Phosphine induced inhibition of mitochondrial cytochrome $\mathrm{c}$ oxidase leads to the generation of reactive oxygen species and cellular peroxides (Chaudhry and Rai 2014). The imbalance between free radicals production and elimination exhibits an oxidative stress that results in lipid peroxidation and oxidant mechanisms with subsequent biological macromolecular damage especially cell membranes ultimately leading to cell death (Mirakbari 2015);(Yousef, Soliman et al. 2015). The effect of phosphine on the cellular mechanisms has been postulated by three ways: a) inhibition of acetylcholinesterase, $b$ ) inhibition of peroxidase and/or c) inhibition of glycerol phosphate dehydrogenase.

Oxidative stress outcomes from an unbalance between radical production and radical-scavenging systems with increased generating of reactive oxygen species (ROS) or minimized activity of antioxidant defenses or both (Maritim, Sanders et al. 2003). Free 
radicals are necessary for any biochemical process and are continuously produced in the body. Contact to pesticides leads to the production of (ROS) which encourages oxidative stress and modulation in antioxidant defense mechanisms (Bhatti, Kiran et al. 2010). Cells have many ways to reduce the harmful effects of oxidative stress, either by restoring an injury or by direct reduction in the occurrence of oxidation by enzymatic and non-enzymatic antioxidants. These include nutritional flavonoids, $\alpha$-lipoic acid, vitamins, and melatonin. However, the quantities of these protective devices present under normal physiological conditions are sufficient only to keep pace with the natural threshold of the physiological rate of free radical generation. Therefore, any additional load of free radicals, whether from an indigenous or exogenous source leads to the oxidative disparity which leads to oxidative stress (Tiwari 2004). AlP reacts with the presence of hydrochloric acid or water in the stomach by releasing the fatal phosphine gas. Animal studies indicate that it works by inhibiting cytochrome oxidase in the mitochondria (Anand, Binukumar et al. 2011);(Moghadamnia 2012). Furthermore, it is shown that AIP increases ROS levels by disrupting the electron transport chain (ETC) which leads to overproduction of free radicals with some modifications in the antioxidant mechanisms (Anand, Sharma et al. 2013),(Kariman, Heydari et al. 2012),(Tehrani, Halvaie et al. 2013).

The toxic appearance caused by different pesticides may be connected with the enhanced production of free radicals. Assess the extent of lipid peroxidation indirectly reflects the degree to which cell membranes are injury by free radicals. MDA is a helpful indicator of the degree of lipid peroxidation. Data in the present study showed clearly that, rats treated with ALP, represented a significant increase in MDA content of the kidney tissue and this could possibly be due to the generation of free radicals following ALP exposure.
$\mathrm{H}_{2} \mathrm{O}_{2}$ overload leads to an increase the level of MDA, protein denaturation, lipid peroxidation in cell membranes (Anand, Binukumar et al. 2011). However, rats that received both ALP and melatonin resulted in significant $(\mathrm{P}<0.05)$ reductions in $\mathrm{MDA}$ in kidney tissues as compared with that of ALP treated rats. This may be due to the strength of melatonin in scavenging the free radicals that are generated during the oxidation of unsaturated lipids and causing the spread of lipid peroxidation (Gilad, Cuzzocrea et al. 1997). That melatonin causes significant inhibition of MDA production and MPO probably in part by scavenging the very reactive $\mathrm{OH}$ and lipid peroxyl radicals (ROO).

In tissues, SOD and CAT are the first line of defense and detection of the toxic effects of ROS (Farombi, Adedara et al. 2014) and have been reported to exhibit synergism in their functioning (Kono and Fridovich 1982) Superoxide radicals are converted to $\mathrm{H}_{2} \mathrm{O}_{2}$ by SOD which is subsequently converted into water and oxygen by the action of CAT. In the present study, it was observed that rats were administered ALP exhibit a significant $(\mathrm{P}<0.05)$ decrease in the activities of the enzymatic antioxidants (SOD, CAT, and GPx), except for glutathione reductase (GR) which shows insignificant $(\mathrm{P}>0.05)$ decrease activities indicated a breakdown of the enzymatic antioxidant system against ALP-induced oxidative stress. (Iyanda and Adeomi 2013), mentioned that phosphine caused cytotoxicity via inhibiting SOD activity and by affecting cellular antioxidant defense. In accordance with our results, (Mehrpour, Jafarzadeh et al. 2012) who reported that AlP causes cellular toxicity via reduction of SOD, CAT and GPx and glutathione activities. In the current study, administration both ALP and melatonin (GIV) showed that melatonin administration increases the activities of GPX, SOD, and CAT in kidney tissue. It was also found that melatonin participates in the recycling of glutathione (GSH) and the preservation a high proportion of GSH / GSSG by facilitating the genetic 
expression of GPX, SOD and catalase, and promoting de novo synthesis of GSH by stimulating the activity of synthetase gl-glutamyl-cysteine (Escames, López et al. 2010). In accordance with the results of Mehdi (Goudarzi, Khodayar et al. 2017) who showed that pretreatment with MEL especially at the dose of 20 $\mathrm{mg} / \mathrm{kg}$ for five consecutive days increases the antioxidative defense such as GSH, GPx, and CAT. Moreover, MEL, at the doses of $20 \mathrm{mg} / \mathrm{kg}$, increases the activity of SOD. Thus, melatonin treatments effectively protected the kidney tissues against oxidative damage.

Increased signs of oxidative stress can cause morphological and functional changes in the kidneys (Quirós, Blanco-Gozalo et al. 2016). The oral administration of ALP induced marked kidney damage in the rat. Histopathological data showed, structural alterations indicated by total loss of normal kidney architecture, these changes were apparent in both Malpighian corpuscle with degenerated and congested glomeruli with hypercellularity and renal tubules. Data of the present study are in agreement with the results of (Mehrpour, Dolati et al. 2008), they reported that histopathological changes are common in AlP fatal poisoning. In accordance with the results reported previously by (Ghazi 2013), who reported that varying degrees of edema, congestion, and leukocytic infiltration, in the kidney after exposure to AlP. The protective effects of MEL on kidney status were confirmed by histopathological studies, revealed almost normal kidney architecture. Such an effect was remarkable in both renal corpuscle and renal tubules. Previous studies have demonstrated the nephroprotective effect of MEL in different pathological conditions. (Manda and Bhatia 2003).

Ultrastructurally, rats ALP in the group (GIII) the renal corpuscle showed obvious signs of injury; glomerular capillaries filled with RBCs, endothelial and mesangial cells deteriorated and increased mesangium matrix. The thickness of the filtration membrane deformed podocytes. Furthermore, it was noticed that the "PCT" appeared highly altered; with disorganized cells and indistinct cell margins; disoriented microvilli, endocytic vesicles. Ruptured mitochondria, unrecognizable ER, increase in the number of lysosomes \& lipid droplets. Moreover, many studies assessed the apoptotic effects of phosphine (Anand, Sharma et al. 2013). The present study showed also, that Kidney sections from rats that received both ALP and melatonin, revealed almost normal kidney architecture. Such an effect was remarkable in both renal corpuscle and renal tubules. Data of the present study confirmed those findings reported by (El-Sokkary and Awadalla 2011) who demonstrated that melatonin administration counteracts all changes induced by cadmium and the tissue appeared more or less normal. Also, it has been shown that MEL is able to restrain kidney failure induced by ciprofloxacin in rats by inhibiting inflammation and oxidative damage (Shaki, Ashari et al. 2016). Moreover, MEL improves diabetic nephropathy in rats' kidneys by reducing oxidative stress (Kurcer, Parlakpinar et al. 2007).

Present data was moreover supported by the immunohistochemical results of the expression of p53 protein. p53, although known as a tumor inhibitor, it quickly responds to cellular stress in both cancer and normal cells. Depending on the severity of the tension, p53 may lead to cell cycle cessation and/or cell death under different physiological conditions. Mechanically, p53 is a major regulator of gene expression in the nucleus. However, p53 may also participate in cell regulation by mechanisms independent of transcription in the cytoplasm (Saifudeen, Dipp et al. 2002). P53 regulates proapoptotic genes, which lead to an increase in the permeabilization of the mitochondrial membrane and expression of apoptogenic genes (Seth, Yang et al. 
2005). ALP damages DNA directly by binding to DNA and/or indirectly by way of oxidative stress, which occurs as a consequence of the accumulation of free radicals, especially hydroxyl radicals (Hsu, Chi et al. 2002). Thus, the activation of two important protein kinases, ATR (ataxia telangiectasia and Rad3-related protein) and ATM (ataxia telangiectasia mutated), are responsible for DNA damage and directly induce phosphorylation and activation of p53 (Shiloh 2003) Similarly, Data in the present study showed clearly that, a relative higher p53 expressions in renal glomeruli and tubular cells after exposure to ALP.

In the current study, administration both ALP and melatonin showed that melatonin administration decreases the activity of p53 in kidney tissue. Since p53 is a tumor suppressor gene involved in the regulation of gene transcription, DNA repair, and triggered apoptosis. Thus, we can suggest that melatonin disassembled ROS and apoptosis, thereby reducing the oxidative stress that eventually activates p53, respectively.

The use of melatonin ameliorated these toxic effects. Metabolism of xenobiotics leads to an increase in the production of electrophilic radicals that leads to the elevation of ROS in cells, increasing free radicals like $\mathrm{H} 2 \mathrm{O} 2$, hydroxyl radicals, singlet oxygen, and peroxynitrite. The spread of ROS is countered by the antioxidant defense mechanism in cells, enzymatic and non-enzymatic, which tends to convert these free radicals into non-toxic products (Ďuračková 2007). melatonin does not subject to redox cycling, once oxidized; it cannot be reduced to its previous state as it forms several stable end-products when interacting with free radicals (Tan, Manchester et al. 2000). Melatonin exercises its antioxidative effects by three different mechanisms: 1 . The results of studies on phospholipid-containing vesicles illustrate that melatonin is found near the polar heads of these molecules in membrane lipid layers (Reiter, Tan et al.
2014). Such specific positioning of melatonin gives this molecule a greater chance of protecting phospholipids against free radicals. 2. Melatonin can easily penetrate into subcellular compartments especially in the mitochondria because of its small size and amphiphilic properties. It affects mitochondrial homeostasis by several mechanisms, finally leading to improving the activity of ETC (Leon, AcuñaCastroviejo et al. 2005).

3. Melatonin has also been found to be a powerful peroxyl radical (LOO·) scavenger. the melatonin metabolites, c3OHM and AMK, were presented as a highly effective LOO scavenger (Mekhloufi, Vitrac et al. 2007, Marchetti, Sidahmed-Adrar et al. 2011). Also, Melatonin also neutralizes hydrogen peroxide, singlet oxygen, peroxynitrite anion, nitric oxide and hypochlorous acid (Reiter 2000). Moreover, it catalyzes many antioxidative enzymes such as superoxide dismutase, GSH peroxidase and GSH reductase which increase its efficiency as an antioxidant (Cuzzocrea, Mazzon et al. 2001).

\section{CONCLUSION}

From the current results, it can be extrapolating the administration of AlP resulted in considerable changes in biochemical and histopathological parameters reflecting renal damage. The present study demonstrated that most of these changes were reversed and minimize by the treatment of melatonin. Thus, we can conclude that melatonin has significant nephroprotective activity against AlP induced nephrotoxicity.

\section{REFRENCES:}

Ahmadiasl, N., et al. (2014). "The anti-inflammatory effect of erythropoietin and melatonin on renal ischemia reperfusion injury in male rats." $\underline{\text { Advanced }}$ pharmaceutical bulletin 4(1): 49.

Anand, R., et al. (2011). "Aluminum phosphide poisoning: an unsolved riddle." Journal of applied toxicology 31(6): 499-505. 
Anand, R., et al. (2013). "Mitochondrial electron transport chain complexes, catalase and markers of oxidative stress in platelets of patients with severe aluminum phosphide poisoning." Human \& experimental toxicology 32(8): 807-816.

Asghari, M. H., et al. (2017). "On the mechanisms of melatonin in protection of aluminum phosphide cardiotoxicity." Archives of toxicology 91(9): 31093120 .

Bancroft, J. D., et al. (1994). Manual of histological techniques and their diagnostic application. Edinburgh

; New York : Churchill Livingstone.

Bhatti, G., et al. (2010). "Modulation of ethion-induced hepatotoxicity and oxidative stress by vitamin $\mathrm{E}$ supplementation in male Wistar rats." Pesticide biochemistry and physiology 98(1): 26-32.

Bhatti, G., et al. (2014). "Ameliorative role of melatonin against cypermethrin induced hepatotoxicity and impaired antioxidant defense system in Wistar rats." IOSR Journal of Environmental Science, Toxicol Food Technol (IOSRJESTFT) 8(1): 39-48.

Bingham, E., et al. (2001). Patty's toxicology. Volume 2: toxicological issues related to metals, neurotoxicology and radiation metals and metal compounds, John Wiley and Sons.

Brod, J. and J. H. Sirota (1948). "The renal clearance of endogenous "creatinine" in man." The Journal of clinical investigation 27(5): 645-654.

Chaudhry, D. and A. S. Rai (2014). "N-acetyl cysteine in aluminum phosphide poisoning: Myth or hope." Indian journal of critical care medicine: peer-reviewed, official publication of Indian Society of Critical Care Medicine 18(10): 646.
Cuzzocrea, S., et al. (2001). "Melatonin reduces dinitrobenzene sulfonic acid-induced colitis." Journal of pineal research 30(1): 1-12.

Detanico, B. C., et al. (2009). "Antidepressant-like effects of melatonin in the mouse chronic mild stress model." European journal of pharmacology 607(1-3): 121-125.

Draper, H. and M. Hadley (1990). [43] Malondialdehyde determination as index of lipid Peroxidation. Methods in enzymology, Elsevier. 186: 421-431.

Dua, R., et al. (2010). "Altered glucose homeostasis in response to aluminium phosphide induced cellular oxygen deficit in rat."

Ďuračková, Z. (2007). "Oxidants, antioxidants and redox stress." The Activity of Natural Compounds in Diseases Prevention and Therapy: 11-59.

Eddleston, M., et al. (2002). "Pesticide poisoning in the developing world - a minimum pesticides list." The Lancet 360(9340): 1163-1167.

Ejaz, S., et al. (2004). "Endocrine disrupting pesticides: a leading cause of cancer among rural people in Pakistan." Exp Oncol 26(2): 98-105.

El-Sokkary, G. H. and E. A. Awadalla (2011). "The protective role of vitamin $\mathrm{C}$ against cerebral and pulmonary damage induced by cadmium chloride in male adult albino rat." The Open Neuroendocrinology Journal 4(1).

Escames, G., et al. (2010). "The role of mitochondria in brain aging and the effects of melatonin." Current neuropharmacology 8(3): 182-193.

Farombi, E., et al. (2014). "Sperm characteristics, antioxidant status and hormonal profile in rats treated with artemisinin." Andrologia 46(8): 893-901. 
Ghazi, M. A. (2013). "WHEAT PILL (ALUMINUM PHOSPHIDE) POISONING"." The Professional Medical Journal 20(06): 855-863.

Gilad, E., et al. (1997). "Melatonin is a scavenger of peroxynitrite." Life sciences 60(10): PL169-PL174.

Goldberg D. and R. Spooner (1983). In Methods of Enzymatic Analysis

(Bergmeyen, H.V. Ed. ) 3rd edn. Vol 3, pp 258 - 265, Verlog Chemie, Deerfield beach, FI.

Goudarzi, M., et al. (2017). "Pretreatment with melatonin protects against cyclophosphamide-induced oxidative stress and renal damage in mice." Fundamental \& clinical pharmacology 31(6): 625-635.

Govender, J., et al. (2014). "Mitochondrial catastrophe during doxorubicin-induced cardiotoxicity: a review of the protective role of melatonin." Journal of pineal research 57(4): 367-380.

Gunnell, D., et al. (2007). "The global distribution of fatal pesticide self-poisoning: systematic review." BMC public health 7(1): 357.

Gurjar, M., et al. (2011). "Managing aluminum phosphide poisonings." Journal of Emergencies, Trauma and Shock 4(3): 378.

Hsu, C. H., et al. (2002). "Melatonin reduces phosphine-induced lipid and DNA oxidation in vitro and in vivo in rat brain." Journal of pineal research 32(1): 53-58.

Iyanda, A. A. and O. A. Adeomi (2013). "Oxidants and Antioxidants in Medical Science."

Kariman, H., et al. (2012). "Aluminium phosphide poisoning and oxidative stress." Journal of Medical Toxicology 8(3): 281-284.

Khurana, V., et al. (2009). "Microangiopathic hemolytic anemia following disseminated intravascular coagulation in aluminum phosphide poisoning."
Kilic, U., et al. (2013). "Melatonin suppresses cisplatininduced nephrotoxicity via activation of $\mathrm{Nrf}-2 / \mathrm{HO}-1$ pathway." Nutrition \& metabolism 10(1): 7.

Kolli, V. K., et al. (2013). "Preclinical efficacy of melatonin to reduce methotrexate-induced oxidative stress and small intestinal damage in rats." Digestive diseases and sciences 58(4): 959-969.

Kono, Y. and I. Fridovich (1982). "Superoxide radical inhibits catalase." Journal of Biological Chemistry 257(10): 5751-5754.

Kurcer, Z., et al. (2007). "Protective effects of chronic melatonin treatment against renal ischemia/reperfusion injury in streptozotocin-induced diabetic rats." Experimental and clinical endocrinology \& diabetes 115(06): 365-371.

Leon, J., et al. (2005). "Melatonin mitigates mitochondrial malfunction." Journal of pineal research 38(1): 1-9.

Manda, K. and A. Bhatia (2003). "Prophylactic action of melatonin against cyclophosphamide-induced oxidative stress in mice." Cell biology and toxicology 19(6): 367-372.

Marchetti, C., et al. (2011). "Melatonin protects PLPC liposomes and LDL towards radical-induced oxidation." Journal of pineal research 51(3): 286-296.

Maritim, A., et al. (2003). "Diabetes, oxidative stress, and antioxidants: a review." Journal of biochemical and molecular toxicology 17(1): 24-38.

Marklund, S. and G. Marklund (1974). "Involvement of the superoxide anion radical in the autoxidation of pyrogallol and a convenient assay for superoxide dismutase." European journal of biochemistry 47(3): 469-474. 
Mehrpour, O., et al. (2008). "Evaluation of histopathological changes in fatal aluminum phosphide poisoning." Indian Journal of Forensic Medicine \&Toxicology 2(2): 34-36.

Mehrpour, O., et al. (2012). "A systematic review of aluminium phosphide poisoning." Archives of Industrial Hygiene and Toxicology 63(1): 61-73.

Mekhloufi, J., et al. (2007). "Quantification of the water/lipid affinity of melatonin and a pinoline derivative in lipid models." Journal of pineal research 42(4): 330-337.

Mirakbari, S. M. (2015). "Hot charcoal vomitus in aluminum phosphide poisoning-A case report of internal thermal reaction in aluminum phosphide poisoning and review of literature." Indian journal of anaesthesia 59(7): 433.

Moghadamnia, A. A. (2012). "An update on toxicology of aluminum phosphide." DARU journal of Pharmaceutical Sciences 20(1): 25.

Paglia D. and W. Valentine (1967). Studies on the quantitative and qualitative characterization of erythrocyte glutathione peroxidase. The Journal of laboratory and clinical medicine 70(1):158-

Parmar, M. Y., et al. (2009). "Hepatoprotective activity of Amomum subulatum Roxb against ethanol-induced liver damage." International Journal of Green Pharmacy (IJGP) 3(3).

Prioleau, J. and S. J. Schnitt (1995). "p53 antigen loss in stored paraffin slides." New England Journal of Medicine 332(22): 1521-1522.

Quirós, Y., et al. (2016). "Cardiotrophin-1 therapy prevents gentamicin-induced nephrotoxicity in rats." Pharmacological research 107: 137-146.

Rahbar Taramsari, M., et al. (2013). "Alteration in liver enzymes in aluminum phosphide poisoning, a retrospective study." Iranian Journal of Toxicology 7(21): 854-857.
Reiter, R. (2000). "Melatonin: mechanisms and actions as an antioxidant." Curr Topics Biophy. 24: 171-183.

Reiter, R. J., et al. (2014). "Melatonin: exceeding expectations." Physiology 29(5): 325-333.

Romero, A., et al. (2014). "A review of metal-catalyzed molecular damage: protection by melatonin." Journal of pineal research 56(4): 343-370.

Saif, Q., et al. (2015). "Aluminium phosphide induced acute kidney injury." The Egyptian Journal of Internal Medicine 27(3): 115.

Saifudeen, Z., et al. (2002). "A role for p53 in terminal epithelial cell differentiation." The Journal of clinical investigation 109(8): 1021-1030.

Seth, R., et al. (2005). "p53-dependent caspase-2 activation in mitochondrial release of apoptosisinducing factor and its role in renal tubular epithelial cell injury." Journal of Biological Chemistry 280(35): 31230-31239.

Shaki, F., et al. (2016). "Melatonin can attenuate ciprofloxacin induced nephrotoxicity: Involvement of nitric oxide and TNF- $\alpha . "$ Biomedicine \& Pharmacotherapy 84: 1172-1178.

Shiloh, Y. (2003). "ATM and related protein kinases: safeguarding genome integrity." Nature Reviews Cancer 3(3): 155-168.

Sinha, A. K. (1972). "Colorimetric assay of catalase." Analytical biochemistry 47(2): 389-394.

Strate, T., et al. (2005). "Microcirculatory function and tissue damage is improved after therapeutic injection of bovine hemoglobin in severe acute rodent pancreatitis." Pancreas 30(3): 254-259.

Subramaniam, K. and J. Solomon (2006). "Organochlorine pesticides BHC and DDE in human 
blood in and around Madurai, India." Indian Journal of Clinical Biochemistry 21(2): 169.

Sudakin, D. (2005). "Occupational exposure to aluminium phosphide and phosphine gas? A suspected case report and review of the literature." Human \& experimental toxicology 24(1): 27-33.

Tabacco, A., et al. (1979). "Simplified enzymic/colorimetric serum urea nitrogen determination." Clinical chemistry 25(2): 336-337.

Tan, D.-X., et al. (2000). "Significance of melatonin in antioxidative defense system: reactions and products." Neurosignals 9(3-4): 137-159.

Tehrani, H., et al. (2013). "Protective effects of Nacetylcysteine on aluminum phosphide-induced oxidative stress in acute human poisoning." Clinical Toxicology 51(1): 23-28.
Tiwari, A. K. (2004). "Antioxidants: new-generation therapeutic base for treatment of polygenic disorders." Current science: $1092-1102$.

Tripathi, S. and S. Pandey (2007). "The effect of aluminium phosphide on the human brain: a histological study." Medicine, science and the law 47(2): 141-146.

Wolden-Hanson, T., et al. (2000). "Daily melatonin administration to middle-aged male rats suppresses body weight, intraabdominal adiposity, and plasma leptin and insulin independent of food intake and total body fat." Endocrinology 141(2): 487-497.

Yousef, M. I., et al. (2015). Aluminium phosphideinduced hepato-nephrotoxicity and oxidative damage in rats: the protective effect of $\alpha$-lipoic acid. The Open conference proceedings journal. 